\title{
Case of post tubectomy tubal partial molar pregnancy
}

\author{
Veena Vidyasagar ${ }^{1, *}$, Preethi E. ${ }^{2}$ \\ ${ }^{\mathbf{1}}$ Associate Professor, ${ }^{2}$ Tutor, ${ }^{1}$ Dept. of Obstetrics and Gynecology, ${ }^{\mathbf{2} D e p t}$. of Pathology, East Point College of Medical Sciences \\ and Research, Bidarhalli, Bangalore, Karnataka, India
}

*Corresponding Author: Veena Vidyasagar

Email: vinav2002@yahoo.co.in

Received: $8^{\text {th }}$ September, 2018

Accepted: $30^{\text {th }}$ October, 2018

\begin{abstract}
A 34 year old lady reported to casualty OPD with complaints of pain abdomen of one day's duration. She gave H/O irregular menstrual periods and had her last menstrual period one month back. Obstetric history $-\mathrm{P}_{2} \mathrm{~L}_{2} \mathrm{~A}_{2}$. MTP and abdominal tubectomy done 7 years back. On per abdominal examination, there was tenderness all over abdomen. Patient was admitted with provisional diagnosis of UTI with APD. Antibiotics and other supportive measures were started. Relevant investigations were ordered for. Patient was referred to Gynaecologist for $\mathrm{C} / \mathrm{O}$ pain lower abdomen and irregular menstrual periods. On per abdominal examination done by Gynaecologist, there was mild rigidity over right side of umbilical region. Urine pregnancy test was weakly positive. Ultrasound revealed mobile internal echoes in urinary bladder, minimal fluid in endometrial cavity and Pouch of Douglas. Serum Beta HCG was sent.

Patient's general condition remained stable. Review scan done on next day revealed features suggestive of right adnexal ectopic gestation with minimal fluid in pelvis. It was decided to take her up for laparotomy.

Abdomen was opened by Pfannesteil incision. Omentum was partially adherent to parietal peritoneum. Blood clot with some tissue (? Products of conception) was seen adjacent to fimbria of right fallopian tube. Same removed along with right sided salpingooopherectomy and left sided tubectomy. Beta hCG levels after surgery showed decreasing trend and remained normal when done 70 days after surgery. Histopathology report of right fallopian tube along with some tissue fragments was suggestive of Partial hydatidiform mole.
\end{abstract}

Keywords: Partial hydatidiform mole, Beta HCG, Molar ectopic pregnancy, Post tubectomy tubal pregnancy.

\section{Introduction}

The incidence of partial or complete hydatidiform mole is approximately one in 500-1000 pregnancies. ${ }^{1}$ Molar pregnancy is mostly seen within the uterus and is very rarely found at ectopic location. ${ }^{2}$ The incidence of ectopic pregnancy is $1-2 \%$ and about $98 \%$ of them occur in fallopian tube. Thus, the probability of a molar ectopic pregnancy would be the incidence of ectopic pregnancy (0.02) multiplied by the incidence of molar pregnancy (0.001). Incidence of tubal ectopic hydatidiform mole is rare and is usually over diagnosed. ${ }^{3}$ Partial mole is mostly seen in women who use oral contraceptives and have irregular menstruation. ${ }^{4}$

In this case report, a woman having obstetric history of 2 normal deliveries and 2 abortions presented with $\mathrm{C} / \mathrm{O}$ pain abdomen. She had undergone abdominal tubectomy 7 yes back. Right tubal pregnancy was diagnosed on the basis of ultrasound and Beta HCG reports. Laparotomy was performed. Right sided salpingooopherectomy and left sided salpingectomy was done. After the surgery, pathological examination revealed partial tubal molar pregnancy.

\section{Case Report}

A 34 year old lady reported to casualty OPD with complaints of nausea for few days and severe pain abdomen for one day. The pain was throbbing in character and non radiating. There was no H/O fever or vomiting. Bowel and bladder habits were regular.
Menstrual History: 3-4/20-30 days irregular normal flow. Date of last menstrual period was one month back. She was a known case of urolithiasis and was on conservative management for it. There was no other significant past medical and surgical history.

On General Examination: Patient was obese. Fully conscious and well oriented. Pulse - 84 bpm. BP -140/70 $\mathrm{mmHg}$. Afebrile. No pallor, icterus, cyanosis. On abdominal examination: Abdomen was soft. There was tenderness all over abdomen. No organomegaly. No free fluid. CVS, Respiratory system, CNS - NAD

Provisional diagnosis of UTI with APD was made by Casualty doctor and patient was admitted in Female surgical ward. She was kept nil orally, IV fluids were transfused, Inj. Ceftriaxone and other supportive measures were started. Relevant investigations were ordered for.

Patient was referred to Gynaecologist for $\mathrm{C} / \mathrm{O}$ pain abdomen and irregular menstrual periods. She gave H/O scanty regular menstrual periods (lasting for one day) for the last one year. She was not sure of date of last menstrual period. Obstetric history $-\mathrm{P}_{2} \mathrm{~L}_{2} \mathrm{~A}_{2}$. She had 2 normal deliveries and 2 first trimester MTPs. Last delivery was 12 years back and MTP and abdominal tubectomy was done 7 years back.

On abdominal examination there was soft abdominal distension with mild rigidity over right side of umbilical region. There were occasional bowel 
sounds. Urine pregnancy test was weakly positive after 5 minutes.

Ultrasound revealed mobile internal echoes in urinary bladder. Uterus was of normal size. Both ovaries were normal. There was minimal fluid in endometrial cavity and Pouch of Douglas. Serum Beta HCG was advised. Patient was transferred to Gynae ward. Most of the laboratory investigations done at the time of admission were normal except for evidence of mild anaemia $(\mathrm{Hb}-8.7 \mathrm{gms} \%$ and $\mathrm{PCV}-28.5 \%)$.

Patient's general condition remained stable. Review scan was done by Senior Radiologist on the next day. It revealed an ill defined echogenic lesion in the right adnexa measuring $5.7 \mathrm{~cm} \mathrm{x} 4.0 \mathrm{~cm}$ demonstrating mild vascularity. Features were suggestive of ectopic gestation in right adnexa with minimal haemorrhagic fluid in pelvis. It was decided to take up patient for laparotomy. Serum Beta hCG report obtained just before surgery was $5351 \mathrm{mIU} / \mathrm{ml}$.

Abdomen was opened by Pfannesteil incision. Omentum was partially adherent to parietal peritoneum. Same separated. There was altered blood in peritoneal cavity. Blood clot with some tissue (? Products of conception) of approximately $5 \times 5.5 \mathrm{~cm}$ seen adjacent to fimbria of right fallopian tube. Same removed along with right sided salpingooopherectomy. Left adnexa appeared normal. Left sided tubectomy done. This was followed by D\&C. Postoperative period was uneventful.

Beta hCG done one week after surgery was 70.19 $\mathrm{miu} / \mathrm{ml}$. It dropped down to $06.81 \mathrm{miu} / \mathrm{ml}$ after 2 weeks and was $0.5 \mathrm{mIU} / \mathrm{ml}$ after 70 days. Patient didn't report for follow up thereafter.

\section{Histopathology Report}

Gross: Received specimen consisting of portion of right fallopian tube along with grey brown tissue fragments. Right tube measures $4 \mathrm{~cm}$, cut surface shows haemorrhagic areas. Multiple grey brown tissue fragments ranging in size from 0.5 to $2 \mathrm{~cm}$. Also received a portion of Left tube measuring $1.3 \mathrm{~cm}$ in length, cut surface shows a patent lumen.

? Products of Conception: Microscopy showed fragments of chorionic tissue comprising of mixed population of normal sized and enlarged hydropic villi. Villi lined by inner cyto trophoblast and outer syncytiotrophoblasts. Enlarged villi are avascular and show central acellular cistern formation. Few of the villi show irregular scalloped outlines. The trophoblastic proliferation is polar in distribution with syncytiotrophoblastic protrusions. Areas of fibrin deposition and organising blood clot are also noted. Few of the normal sized villi show fibrosis. No fetal parts are identified. Overall morphological features are suggestive of Partial hydatidiform mole.

Right Fallopian Tube: Mucosa lined by ciliated columnar epithelium. Lamina propria shows vascular connective tissue and haemorrhage. Wall of the tube shows focal areas of fibrinoid necrosis and haemorrhage.
Interspersed areas of decidualisation and scattered cytotrophoblsst and syncytio trophoblasts. Adjacent area shows a thin walled cyst lined by stratified cuboidal epithelium exhibiting no atypia. Overall morphological features are of ectopic gestation with a paratubal cyst.

Left Fallopian Tube: Normal morphology

Endometrium: Secretory phase with decidualisation

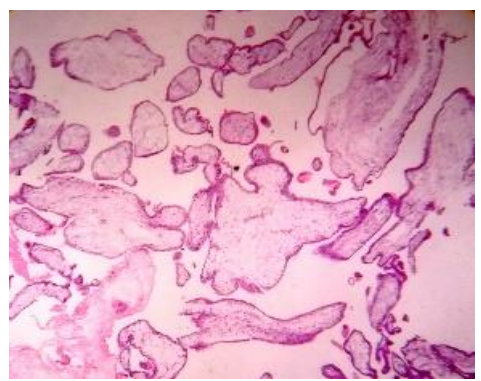

Fig. 1: 4x-Variable sized villi

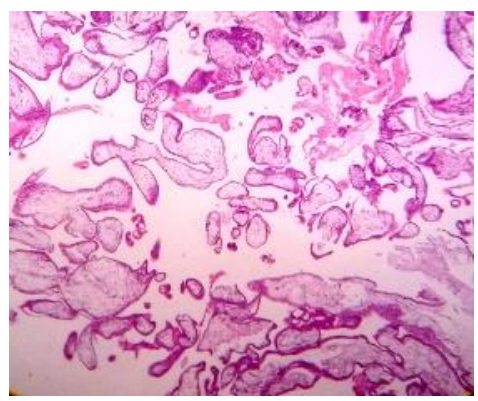

Fig. 2: 10x-Villi showing scalloped outlines

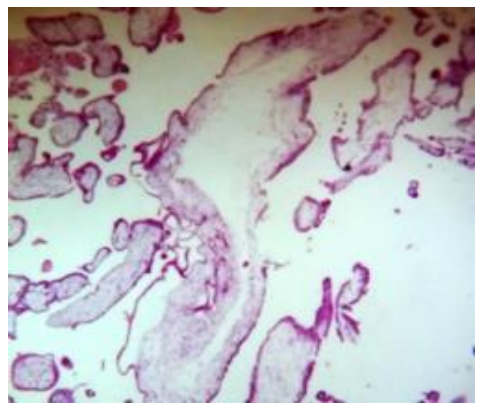

Fig. 3: 40x- Hydropic villi with cisterns

\section{Discussion}

Molar pregnancy arises as a result of abnormal fertilization of ova. The incidence of partial hydatidiform mole has been reported as 1:695 pregnancies. $^{5}$ Theoretically, the proportion of ectopic pregnancies affected by molar change should corroborate with that seen in intrauterine location since the main etiologic factor preceding both partial and complete hydatidiform moles is an abnormal androgenetic chromosomal constitution of the conceptus that is present before implantation, regardless of the site. ${ }^{6}$ Number of cases of partial mole is seen more in women using oral contraceptives and having irregular menstrual cycles. ${ }^{3}$ Though our patient didn't have irregular menstrual periods, as mentioned to the Gynaecologist, she was 
having scanty menstruation for the last one year. Clinical presentation and ultrasound findings of tubal molar pregnancy and tubal pregnancy with normal chorionic tissue are similar. Therefore, it is not possible to differentiate between the two based on clinical presentation and ultrasound picture. Previous small series suggest that it may be difficult to diagnose hydatidiform mole in tubal pregnancies on histopathology. ${ }^{6} 132$ cases with a diagnosis of possible hydatidiform mole in a tubal pregnancy were referred to Charing Cross Hospital between 1986 and 2004. Only two cases were found to be ectopic partial mole. None of the cases developed persistent gestational trophoblastic disease. HCG concentrations returned to normal after few weeks in all cases with confirmed diagnosis of hydatidiform mole. Strict criteria should be established to avoid over diagnosing molar pregnancy and thus avoid unnecessary exposure of patients to chemotherapy.

The diagnosis of hydatidiform mole on histopathology is mainly made on presence of abnormal, nonpolar trophoblast proliferation which is circumferential in nature and often demonstrates a vacuolated phenotype. This may be associated with sheets of pleomorphic extravillus trophoblast fragments. ${ }^{7}$ The extravillus trophoblastic proliferation may appear more florid in ectopic molar pregnancy than in molar pregnancy confined to uterus. Adherence to strict criteria regarding morphologic abnormalities are a must for diagnosis of molar pregnancy. ${ }^{6}$ An ectopic molar pregnancy, at times, may be associated with apparent local invasion of surrounding tissues by the trophoblast. $^{8}$

All pathological specimens of our study case were reviewed and final diagnosis was confirmed. It is not possible to differentiate between molar and non-molar ectopic pregnancies on the basis of B-HCG levels. Beta HCG level increase in our case is comparatively high for an ectopic pregnancy detected at 4 to 5 weeks' gestation. The levels of Beta HCG in molar ectopic pregnancy are usually lower than in molar intrauterine pregnancy because implantation in the fallopian tube may prevent adequate vascularisation. ${ }^{9}$ One cannot differentiate between molar tubal pregnancies and ectopic pregnancy based on B-HCG levels. Previous ectopic pregnancy, pelvic inflammatory disease, tubal ligation, tubal surgery and an intrauterine contraceptive device in situ have been identified as risk factors for ectopic pregnancy. However, $50-75 \%$ of ectopic pregnancies have no obvious risk factors. ${ }^{10}$ Our patient had molar ectopic pregnancy 7 years after abdominal tubectomy.

One cannot differentiate patients with molar tubal pregnancies from those with non-molar tubal pregnancies based on presenting symptoms, signs or laboratory tests. Most common symptoms in tubal gestational trophoblastic disease (GTD) are vaginal bleeding and lower abdominal pain, followed by amenorrhea. $60 \%$ of cases of molar ectopic gestations present as ruptured tubal hydatidiform mole. This presentation is more common in molar ectopic pregnancy as compared to nonmolar ectopic pregnancy. ${ }^{8}$ The present case presented as tubal abortion.

Chances of persistent GTD after an extra-uterine molar gestation is approximately $0.5 \%$ for partial and $15 \%$ for complete hydatidiform moles. Cases of primary tubal choriocarcinoma have been reported. Histopathological diagnosis of such cases poses no specific problems as the features are identical to choriocarcinoma at other sites. ${ }^{8}$

\section{Conclusion}

Ectopic molar pregnancy is a rare condition. It can occur at any location in the pelvic cavity. The clinical features of ectopic molar and non molar pregnancy are similar. Ultrasonography is usually not helpful in diagnosis of ectopic molar pregnancy. Histopathological examination of products of conception is the present gold standard for the diagnosis. ${ }^{11}$ Invasive mole and choriocarcinoma may follow such a pregnancy.

\section{References}

1. Amirian M, Hasanzadeh M, Hafizi L. "Ectopic Partial Molar Pregnancy: A Case Report”. Journal of midwifery and reproductive health. 2017;5(1):848-850.

2. Samaila MO, Adesiyun AG, Bifam C. "Ruptured tubal hydatidiform mole". J Turkey-German Assoc. 2009;10:172-4.

3. Burton JL, Lidbury EA, Gillespie AM, Tidy JA, Smith O, Lawry J, et al. "Over-diagnosis of hydatidiform mole in early tubal ectopic pregnancy”. Histopathology. 2001;38(5):409-417.

4. Heidar Z, Sarfjoo FS, Zademodares S, Habibollahi, M, Kheiri N. Molar Ectopic Pregnancy after Tubectomy Acta Medica Iranica. 2012;50(8):565-567.

5. Beena D, Tirthanath S, Jose V, Shetty J. "Molar pregnancy presents as tubal ectopic pregnancy: a rare case report". Journal of Clinical and Diagnostic Research. 2016;10(1):ED10-ED11.

6. Sebire NJ, Lindsay I, Fisher RA, Savage P, Seckl MJ. Overdiagnosis of complete and partial hydatidiform mole in tubal ectopic pregnancies. International Journal of Gynecological Pathology. 2005;24(3):260-264.

7. Ghazi GF, Donovan PO. Partial mole in ectopic pregnancy. Gynecol Surg. 2006;3:141-143

8. Tanha FD, Shir Ali E. Molar Pregnancy Presents as Tubal Ectopic Pregnancy. Int J Fertil Steril. 2011;4(4):184-186.

9. Chase JS, Check JH, Nowroozi K, Wu CH. First trimester serum levels of the beta-subunit of human chorionic gonadotrophin in a tubal molar pregnancy. Am J Obstet Gynecol. 157:910

10. Patil N, Nazeer RM, Tele J. Case Report Ectopic tubal pregnancy with partial mole: a rare case. Int J Reprod Contracept Obstet Gynecol. 2018;7(4):1651-1653.

11. Borah T, Raphael V, Panda S, Saharia P. Ectopic Molar Pregnancy: A Rare Entity. J Reprod Infertil. 2010;11(3):201-203.

How to cite this article: Vidyasagar V, Preethi E. Case of post tubectomy tubal partial molar pregnancy. Indian $\mathrm{J}$ Obstet Gynecol Res. 2018;5(4):570-572. 